\title{
Cavitation Measurement during Sonic and Ultrasonic Activated Irrigation
}

\author{
Ricardo Macedo, DMD, MSc, ${ }^{*}$ Bram Verbaagen, MSc, PbD, ${ }^{\dagger}$ David Fernandez Rivas, MSc, PbD, ${ }^{\ddagger}$ \\ Michel Versluis, PhD, ${ }^{+}$Paul Wesselink, PhD, ${ }^{*}$ and Luc van der Sluis, DDS, PhD ${ }^{\downarrow}$
}

\begin{abstract}
Ahstract
Introduction: The aims of this study were to quantify and to visualize the possible occurrence of transient cavitation (bubble formation and implosion) during sonic and ultrasonic (UAI) activated irrigation. Methods: The amount of cavitation generated around several endodontic instruments was measured by sonochemiluminescence dosimetry inside 4 root canal models of human dimensions and varying complexity. Furthermore, the spatial distribution of the sonochemiluminescence in the root canal was visualized with long-exposure photography. Results: Instrument oscillation frequency, ultrasonic power, and file taper influenced the occurrence and amount of cavitation. In UAl, cavitation was distributed between the file and the wall extending beyond the file and inside lateral canals/isthmuses. In sonic activated irrigation, no cavitation was detected. Conclusions: Cavitation was shown to occur in UAI at clinically relevant ultrasonic power settings in both straight and curved canals but not around sonically oscillating instruments, driven at their highest frequency. ( $J$ Endod 2014;40:580-583)
\end{abstract}

\section{Key Words}

Activated irrigation, cavitation, sonic, sonochemiluminescence, ultrasonic

From the ${ }^{*}$ Department of Cariology, Endodontology and Pedodontology, ACTA Academic Center for Dentistry Amsterdam, University of Amsterdam and VU University, Amsterdam; ${ }^{\dagger}$ Physics of Fluids Group and MIRA Institute for Biomedical Technology and Technical Medicine and MESA+ Institute for Nanotechnology, University of Twente, Enschede; ${ }^{\ddagger}$ Mesoscale Chemical Systems Group, MESA+ Institute for Nanotechnology, University of Twente, Enschede; and ${ }^{\S}$ Center for Dentistry and Oral Hygiene, University Medical Center Groningen, Groningen, The Netherlands.

Address requests for reprints to $\mathrm{Dr}$ Ricardo Gomes Macedo, University of Amsterdam and VU University, Department of Cariology, Endodontology and Pedodontology, ACTA Academic Center for Dentistry Amsterdam, Gustav Mahlerlaan 3004, 1081LA Amsterdam, The Netherlands. E-mail address: r.macedo@acta.nl 0099-2399/\$ - see front matter

Copyright $\odot 2014$ American Association of Endodontists. http://dx.doi.org/10.1016/j.joen.2013.09.018
Conic activated irrigation (SAI) and ultrasonic activated irrigation (UAI) aim to $\checkmark$ improve the chemical and mechanical efficiency of root canal irrigation procedures (1-4). For both methods, acoustic microstreaming and hydrodynamic cavitation (the formation and implosion of vapor bubbles) have been claimed as their working mechanisms (5-8). However, for SAI, Jiang et al (4) suggested that cavitation cannot occur because the velocity of the sonically oscillating instrument is too low. For UAI, Ahmad et al (9) argued that cavitation is unlikely to occur inside the root canal. Because of space restrictions, the amplitude of oscillation of the endodontic instrument would probably not be high enough to produce cavitation. However, recently cavitation was shown to occur during UAI within the confinement of a root canal $(10,11)$, even with contact of the file against the wall (12), although at higher power settings than advised by the manufacturer for endodontic purposes. To our knowledge, cavitation has never been observed during SAI.

A distinction can be made between transient cavitation, involving a violent inertial collapse of a bubble, and stable cavitation, which involves more gentle volumetric oscillations (13). In this article we investigate specifically the occurrence of transient cavitation involved in enhancing chemical reactions $(7,14,15)$, surface cleaning, and erosion (16).

The aims of this study were to quantify and to visualize the occurrence of cavitation during UAI and SAI in a root canal model of human dimensions with varying morphologic complexity and with different oscillating instruments and power settings. In transient cavitation, high pressure and temperature conditions inside the collapsing bubble lead to the formation of $\mathrm{OH} \bullet$ radicals by sonolysis of $\mathrm{H}_{2} \mathrm{O}$ molecules (14). A chemiluminescent molecule can react with the $\mathrm{OH} \bullet$ radicals and produce light emission (sonochemiluminescence $[\mathrm{SCL}])(17,18)$. No SCL is generated with stable cavitation. Strict dark conditions and sensitive photomultipliers were used here to detect and quantify the production of SCL in cavitation bubbles at various power settings as a direct means to quantify the amount of cavitation. Long-exposure SCL photography provided additional information on the location of cavitation bubbles at different operation and confinement conditions (11).

\section{Materials and Methods}

The methodology includes 2 distinct experiments, sonochemical dosimetry and visualization experiments; both were explained in detail in a previous study (11). Both took place in a light-tight box with dimensions $1.2 \times 1.0 \times 0.5 \mathrm{~m}$; dark conditions inside were verified with long-exposure photography. Inside the box, an endodontic file was positioned in an in-house manufactured polydimethylsiloxane (PDMS) (Sylgard 184; Dow-Corning, Midland, MI) root canal model. The model was of human dimensions (apical diameter, $0.35 \mathrm{~mm}$; taper, $6 \%$; length, $20 \mathrm{~mm}$ ), fixed inside a $1.0 \times 1.0 \times 4.0 \mathrm{~cm}$ cuvette (Plastibrand; Brand, Wertheim, Germany).

SAI was performed by the Endoactivator system (Dentsply, Tulsa, OK) by using the yellow (apical size ISO 15/0.02 taper), red (25/0.04), and blue (35/0.04) polymer tips. The highest operational frequency $(190 \pm 5 \mathrm{~Hz})$ (4) was used because that is most likely to generate cavitation. For UAI, IrriSafe files 25/21 (apical size/length) and 25/ 25 and K25/21 (Satelec Acteon, Merignac, France) were used $(10,18)$, driven with an endodontic ultrasound device (P-Max; Satelec Acteon). The power settings on the 
device ranged from low to high by using colors codes, from Green via Yellow and Blue to Red, each with 10-step increments.

For the sonochemical dosimetry measurements, the instruments were centered and fixed at $1 \mathrm{~mm}$ from working length (WL) by using a translation stage. The ultrasound and sonic devices were switched on and off by a pulse-delay generator (TGP110; TTi, Huntingdon, UK) in 3 cycles, with a period of 10 seconds consisting of 3 seconds ON and 7 seconds OFF (duty cycle of $30 \%$ ). The rest phase in between pulses allows the fluid to relax to its ambient state with respect to its temperature, gas content, and flow. Each measurement was done 6 times with a new file and fresh irrigant. No file/tip separation has been observed during the experiments.

For all SCL experiments, air-saturated aqueous luminol (0.1 $\mathrm{mmol} / \mathrm{L}$ luminol in $0.1 \mathrm{~mol} / \mathrm{L} \mathrm{NaOH}$ ) (Merck Millipore, Billerica, MA) solution was used (19). A photomultiplier tube (PMT) (R508; Hamamatsu Photonics, Hamamatsu, Japan) was placed next to the cuvette. The PMT received an electrical voltage of $1.6 \mathrm{kV}$ from a power supply (6516A; Hewlett-Packard, Palo Alto, CA); its output was recorded at a rate of $300 \mathrm{kHz}$ with a high-speed data acquisition device (USB-6356; National Instruments, Austin, TX). The average and standard deviation of each of the 18 activation cycles (6 measurements of 3 cycles) were calculated as outlined before (11). Calibration showed a linear response of the PMT up to an output voltage of $1 \mathrm{~V}$, above which saturation occurs (underestimation of the amount of cavitation). An interclass correlation coefficient score of 0.994 for single measurements with $P<.001$ was found (11), assuring high reliability and reproducibility.

In the visualization experiments, the spatial distribution of the SCL in the root canal during UAI with a IrriSafe file $25 / 25$ was observed by using long-exposure photographs, which were taken with a CMOS photo camera (D300; Nikon, Tokyo, Japan) and 50-mm, f/1.8 lens (Nikon). The camera exposure time was set at 30 seconds, with highest ISO value (Hi1.0) and automatic white balance. A picture of the root canal model was taken in ambient light before the SCL photos were acquired under dark conditions to identify the location of the root canal model walls.

Four different root canal models were used (Fig. 1), with varying morphologic complexities but equal main root canal size (apical diameter, $0.35 \mathrm{~mm}$; taper, $6 \%$; length, $20 \mathrm{~mm}$ ). These models were made out of PDMS, which has good optical accessibility. The first model was a straight canal; the file was centered and fixed at 2 or $5 \mathrm{~mm}$ from WL. The second model had a lateral canal of $0.250-\mathrm{mm}$ diameter located $3 \mathrm{~mm}$ from WL; the file tip was $2 \mathrm{~mm}$ from $\mathrm{WL}$ and oscillated in the di- rection of the lateral canal. The third model had an isthmus of $3 \times 0.15$ $\times 4 \mathrm{~mm}$ located $2-5 \mathrm{~mm}$ from the apex; the file was placed $2 \mathrm{~mm}$ from WL and oscillated in the direction of the isthmus. The fourth model had an apical curvature, starting $4 \mathrm{~mm}$ from the apex and with a Schneider angle of $27^{\circ}(20)$. The file was inserted either at the start of the curvature ( $4 \mathrm{~mm}$ from $\mathrm{WL})$ or was pre-bent and inserted up to $1 \mathrm{~mm}$ from WL, leading to contact with the root canal wall.

\section{Statistical Analysis}

The Student $t$ tests for independent samples were performed to assess differences in SCL between files with different lengths (21 vs 25 $\mathrm{mm}$ ) and file type (square [K-file] with $2 \%$ taper versus square with round edges [Irrisafe] with $0 \%$ taper). For all tests, $P$ values $<.05$ were considered statistically significant.

\section{Results}

The SCL values for all studied sonic and ultrasonic tips inside the root canal model are plotted in Figure 2. It is evident that cavitation does not occur around sonic tips. An increased power setting increases the SCL signal for all ultrasonic files $(P<.001)$. Already at the power setting of Green 5, a SCL signal could be observed for the Irrisafe files. Both Irrisafe files resulted in a SCL value higher than the K-files of similar dimension $(P=.001)$. The length of the ultrasonic file had no influence on the SCL value $(P>.05)$.

Figure 3 shows the location of cavitation along 2 ultrasonically oscillating files. The IrriSafe file shows 5 locations of cavitation, whereas the K-file only shows cavitation at the tip and the first antinode. These locations appear to match well with the antinodes on the files as obtained in a previous study (21).

Overlaying the long-exposure SCL photos with the ambient light photos of the root canal models with varied morphologic complexity shows the spatial distribution of cavitation (Fig. 1). For all models, cavitation occurred between the ultrasonic file and the wall. SCL was also observed at the entrance of simulated lateral morphologies and beyond the tip of the file, up to approximately $1 \mathrm{~mm}$ when the file was at $\mathrm{WL}-2$ $\mathrm{mm}$ and up to $2 \mathrm{~mm}$ when the file was at $\mathrm{WL}-5 \mathrm{~mm}$ in the straight canal. Cavitation occurred even in curved canal models, with a file oscillating before or beyond the curvature with file-to-wall contact.
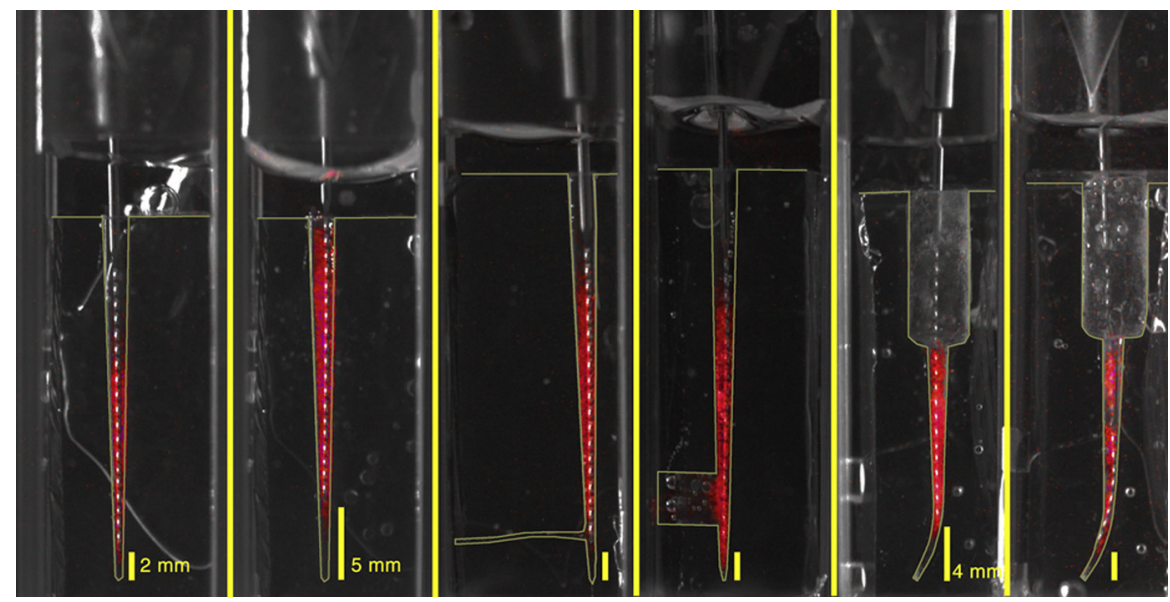

Figure 1. Overlay of SCL photos (represented in red) with ambient light photos, showing the spatial distribution of cavitation inside human-sized root canal models with varied complexity. The confinements have been outlined with thin solid yellow lines; the file insertion depth $(2,4$, or 5 mm) is indicated with yellow bars next to the apical part of the canal. 


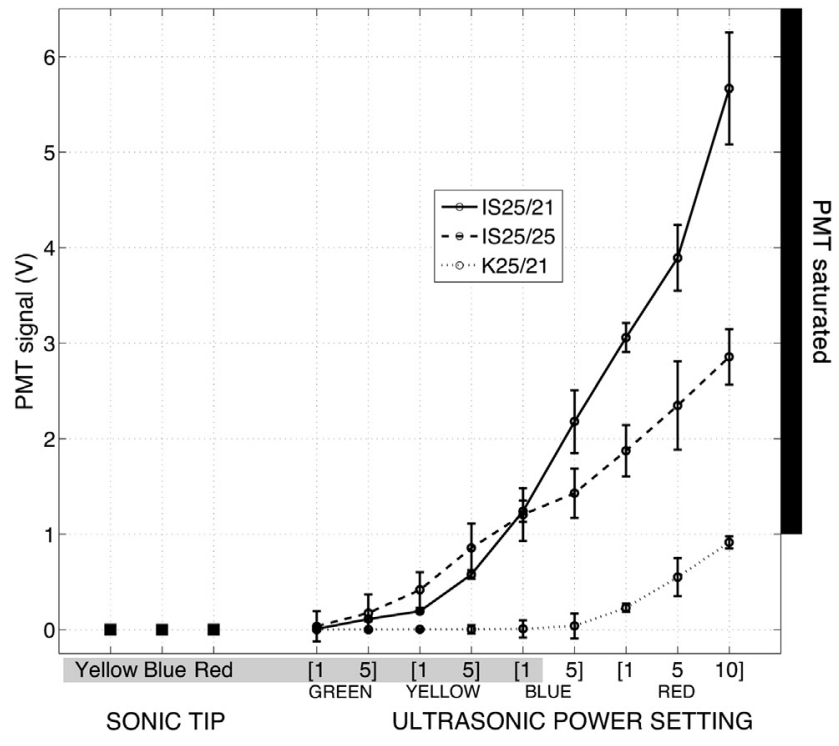

Figure 2. Average and standard deviation of the SCL signal for 3 different ultrasonic files with varying length, diameter, and cross section and for 3 sonic tips. All instruments oscillated inside a human-sized root canal model. The settings marked in gray are clinically relevant (according to the manufacturers). For values above $1 \mathrm{~V}$, the PMT was saturated, and those values are therefore underestimated.

\section{Discussion}

The occurrence of transient cavitation during UAI, although claimed by some $(1,22)$ and doubted by others $(9,23)$, has now been demonstrated in this article for clinically relevant power settings. The reason for such controversy up to now is that cavitation dosimetry in an endodontic context is experimentally challenging and limited by several factors. The most important ones are the small volumes of irrigant used in dentistry $(10-100 \mathrm{~mL})$, the short duration of ultrasonic agitation (20-60 seconds), and the contact of the irrigant with open-air atmospheric conditions. Online dosimetry methods such as SCL have recently been successfully applied in the dental context to quantify transient cavitation occurring around oscillating endodontic files (11) and scalers (6).

The type of file influenced the amount of cavitation (Figs. 2 and 3); the Irrisafe file design was more effective than the K-file with similar dimensions. These 2 files differ in taper ( $2 \%$ for $\mathrm{K}$-file and $0 \%$ for IrriSafe) and cross section (square [K-file] and square with rounded edges [IrriSafe]). The shape of the edges has been proven to have no effect on cavitation generation (11). However, the taper affects the number and amplitude of the antinodes on the file. All the antinodes on the IrriSafe file have an amplitude sufficiently high to induce cavitation, whereas only the tip and the first antinode on the K-file have such a high amplitude (Fig. 3).

By using similar methodology, Ahmad and Roy (24) in 1994 reported limited SCL signal even at high power intensity, making them question the clinical occurrence of cavitation. They suggested that file-to-wall contact would dampen the file oscillation and therefore inhibit the occurrence of cavitation. The comparison of their results with those presented here is difficult, but their negative results may be attributed to the dissipation of energy at the Cavi-endo coupling system (25). In addition, it has been proven in a recent article that unintentional file-to-wall contact occurs in a clinical context but does not completely stop the oscillation of the file, the microstreaming, or cavitation (12).
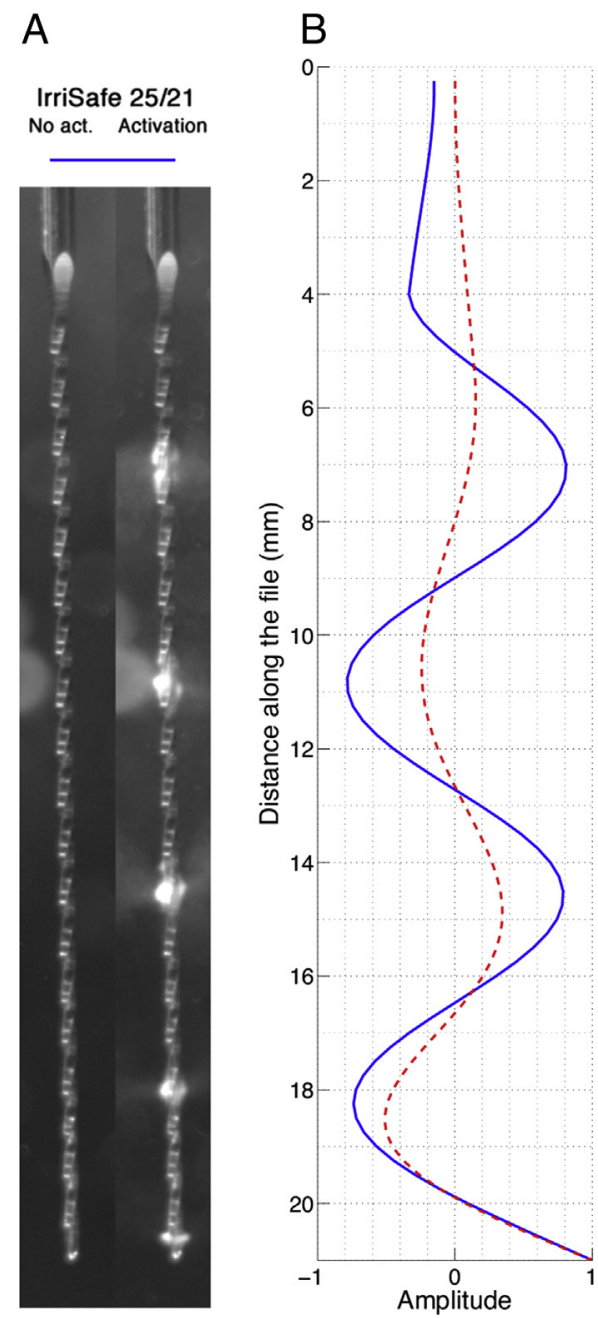

C

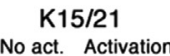

No act. Activation
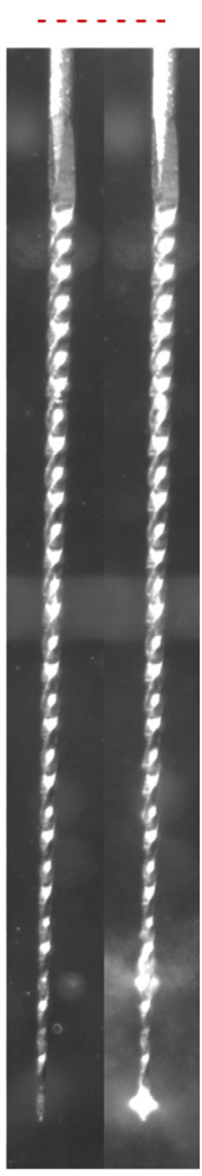

Figure 3. Cavitation occurrence along the IrriSafe $25 / 21(A)$ and K15/21 (C) files during ultrasonic activation in water, as observed with long-exposure photography. The white clouds originate from light reflected off cavitation bubbles. The oscillation patterns of the 2 files (21) are shown in $(B)$ for correlation between the file antinodes and the locations of the cavitation.

The generation of cavitation may be different in a dentin root canal compared with the present PDMS model. However, in previous studies such PDMS models were found to be a suitable root canal model (21), and the change in light transmission has been corrected for (11). The use of luminol solution instead of $\mathrm{NaOCl}$ as irrigant may have underestimated the amount of cavitation produced, because $\mathrm{NaOCl}$ may act as a surfactant and thereby favor cavitation (26). However, luminol is required to detect SCL but cannot be combined with $\mathrm{NaOCl}$ because the 2 react (11). In a recent article (11) more cavitation was detected visually around an endodontic file oscillating in $\mathrm{NaOCl}$ than in luminol. This affects all ultrasonic groups equally.

In this study, SAI at the highest frequency showed no SCL, regardless of the tip size, whereas UAI produced SCL throughout its complete power range. The absence of cavitation during SAI was predicted by Jiang et al (4) because of the reported slow movement of the tip, which was below the threshold needed for cavitation. Such a cavitation threshold can be determined by estimating the pressures required for the formation, growth, and collapse of a bubble within a given liquid. Bubbles can grow when the applied pressure drops from the ambient pressure to below the vapor pressure of the liquid $(27,28)$. Such negative pressures need to be 
generated by the oscillating tip, where the oscillation amplitude is maximal. The likelihood of cavitation occurrence is defined by the cavitation number Ca:

$$
C a=\frac{P_{\text {Atmosferic }}-P_{\text {vapor }}}{\frac{1}{2} \rho U^{2}}
$$

where $\rho$ is the density of the liquid $\left(1100 \mathrm{~kg} / \mathrm{m}^{3}\right.$ for $\left.\mathrm{NaOCl}\right)$. With sufficient cavitation nuclei (preexisting gas pockets) available and for $\mathrm{Ca}<1$, cavitation can occur. The corresponding velocity threshold is $15 \mathrm{~m} / \mathrm{s}$.

The velocity $U$ of the (ultra)sonic tip can be calculated with the equation $U=2 \pi f A$ (with $f$ being the file oscillation frequency and $A$ the amplitude of oscillation). The typical frequency of oscillation for endodontic devices is $30 \mathrm{kHz}$, and with a typical oscillation amplitude of approximately $100 \mu \mathrm{m}$ at high power settings $(21,29,30)$, it exceeds the $\mathrm{Ca}<1$ condition, instantly leading to cavitation. On the other hand, a sonic tip oscillating at a frequency of $190 \mathrm{~Hz}$ and oscillation amplitude of $1.2 \mathrm{~mm}$ oscillates with a velocity of only $1.4 \mathrm{~m} / \mathrm{s}$. Furthermore, the diameter of the apical root canal is smaller than $0.5 \mathrm{~mm}$, which implies extensive wall contact between the tip and the root canal wall for SAI. This inhibits free oscillation of the sonic tip, thereby reducing the velocity of the irrigant $(4,27)$. This was further confirmed by the poor dentin debris removal performance of SAI in simulated oval extensions in an ex vivo root canal model (4).

The long-exposure photography used in this study permits the observation of the spatial distribution of SCL inside root canal models with varied morphologic complexity such as curved main canals and lateral internal morphology (Fig. 1) as often encountered in root canal systems (31). The results of this study show that cavitation is not limited to the ultrasonic file tip but is also distributed further up along the file. These findings corroborate the ones reported by Macedo et al (11) where SCL has been related to a distribution of nodes and antinodes along the file.

SCL has also been observed here in simulated lateral morphology, but only a small distance into the simulated lateral canal and isthmus. This suggests that the cavitation is induced by the oscillating pressure generated when the file oscillates at ultrasonic frequencies. This pressure has recently been shown to extend only a limited distance away from the file (30).

Cavitation was observed up to approximately $2 \mathrm{~mm}$ beyond the tip of the file, even in a curved canal where contact with the wall took place. Such results reinforce the assumption that cavitation is possible under clinical conditions where file-to-wall contact cannot be completely avoided (12). Furthermore, together with a recent article on the flow in curved canals (32), it shows that the presence of a curvature does not significantly affect the mechanisms of action of ultrasonic activation. Future studies should focus on the contribution and optimization of this phenomenon for cleaning the root canal system.

In conclusion, cavitation was shown to occur in UAI at clinically relevant ultrasonic power settings in straight and curved canals, at the entrance of lateral canals and isthmuses, and also up to $2 \mathrm{~mm}$ beyond the tip of the file. No cavitation was detected around sonically oscillating instruments, driven at their highest frequency.

\section{Acknowledgments}

The authors deny any conflicts of interest related to this study.

\section{References}

1. van der Sluis LWM, Versluis M, Wu MK, Wesselink PR. Passive ultrasonic irrigation of the root canal: a review of the literature. Int Endod J 2007;40:415-26.
2. Macedo RG, Wesselink PR, Zaccheo F, et al. Reaction rate of $\mathrm{NaOCl}$ in contact with bovine dentine: effect of activation, exposure time, concentration and $\mathrm{pH}$. Int Endod J 2010;43:1108-15.

3. Moorer WR, Wesselink PR. Factors promoting the tissue dissolving capability of sodium hypochlorite. Int Endod J 1982;15:187-96.

4. Jiang LM, Verhaagen B, Versluis M, van der Sluis LW. Evaluation of a sonic device designed to activate irrigant in the root canal. J Endod 2010;36:143-6.

5. Jiang LM, Verhaagen B, Versluis M, van der Sluis LW. The influence of the oscillation direction of an ultrasonic file on the cleaning efficacy of passive ultrasonic irrigation. J Endod 2010;36:1372-6.

6. Ahmad M, Pitt Ford T, Crum LA. Ultrasonic debridement of root canals: an insight into the mechanisms involved. J Endod 1987;13:93-101.

7. Joyce Tiong T, Price GJ. Ultrasound promoted reaction of rhodamine B with sodium hypochlorite using sonochemical and dental ultrasonic instruments. Ultrason Sonochem 2012;19:358-64.

8. Ruddle CJ. Hydrodynamic disinfection "Tsunami” endodontics. Int Dent SA 2009; 11:6-18.

9. Ahmad M, Pitt Ford T, Crum LA, Walton AJ. Ultrasonic debridement of root canals: acoustic cavitation and its relevance. J Endod 1988;14:486-93.

10. Jiang LM, Verhaagen B, Versluis M, et al. The influence of the ultrasonic intensity on the cleaning efficacy of passive ultrasonic irrigation. J Endod 2011;37:688-92.

11. Macedo RG, Verhaagen B, Fernandez Rivas D, et al. Sonochemical and high-speed optical characterization of cavitation generated by an ultrasonically oscillating dental file in root canal models. Ultrason Sonochem 2014;21:324-35.

12. Boutsioukis $\mathrm{C}$, Verhaagen $\mathrm{B}$, Walmsley $\mathrm{AD}$, et al. Measurement and visualization of file-to-wall contact during ultrasonically activated irrigation in simulated canals. Int Endod J 2013;11:1046-255.

13. Brennen CE. Cavitation and Bubble Dynamics. New York: Oxford University Press; 1995.

14. Suslick K, Doktycz S, Flint E. On the origin of sonoluminescence and sonochemistry. Ultrasonics 1990;28:280-90.

15. Fernandez Rivas D, Prosperetti A, Zijlstra AG, et al. Efficient sonochemistry through microbubbles generated with micromachined surfaces. Angew Chem Int Ed Engl 2010;49:9699-701.

16. Fernandez Rivas D, Verhaagen B, Seddon J, et al. Localized removal of layers of metal, polymer or biomaterial by cavitating microbubbles. Biomicrofluidics 2012; 6:034114.

17. Hatanaka S, Mitome H, Yasui K, Hayashi S. Single-bubble sonochemiluminescence in aqueous luminol solutions. J Am Chem Soc 2002;124:10250-1.

18. Kanthale P, Ashokkumar M, Grieser F. Sonoluminescence, sonochemistry $\left(\mathrm{H}_{2} \mathrm{O}_{2}\right.$ yield) and bubble dynamics: frequency and power effects. Ultrason Sonochem 2008; $15: 143-50$

19. Fernandez Rivas D, Ashokkumar M, Leong T, et al. Sonoluminescence and sonochemiluminescence from a microreactor. Ultrason Sonochem 2012;19:1252-9.

20. Schneider SW. A comparison of canal preparations in straight and curved root canals. Oral Surg 1971;32:271-5.

21. Verhaagen B, Lea SC, van der Sluis LWM, et al. Oscillation characteristics of endodontic files: numerical model and its validation. IEEE Trans Ultrason Ferroelectr Freq Control 2012;59:2448-59.

22. Roy RA, Ahmad M, Crum LA. Physical mechanisms governing the hydrodynamic response of an oscillating ultrasonic file. Int Endod J 1994;27:197-207.

23. Zehnder M. Root canal irrigants. J Endod 2006;32:389-98.

24. Ahmad M, Roy RA. Some observations on the breakage of ultrasonic files driven piezoelectrically. Endod Dent Traumatol 1994;10:71-6.

25. Williams AR, Walmsley AD. Exposimetry of low-frequency ultrasonic dental devices. IEEE Trans Ultrason Ferroelectr Freq Control 1988;35:264-9.

26. Mason TJ, Lorimer JP. Applied Sonochemistry: Uses of Power Ultrasound in Chemistry and Processing. Weinheim, Germany: John Wiley and Sons Ltd - Wiley-VCH Verlag $\mathrm{GmbH} ; 2003$.

27. Marmottant $\mathrm{P}$, Versluis $\mathrm{M}$, de Jong $\mathrm{N}$, et al. High-speed imaging of an ultrasounddriven bubble in contact with a wall: "narcissus" effect and resolved acoustic streaming. Exp Fluids 2006;41:147-53.

28. Liu RH, Yang J, Pindera MZ, et al. Bubble-induced acoustic micromixing. Lab Chip 2002;2:151-7.

29. Lea SC, Walmsley AD, Lumley PJ. Analyzing endosonic root canal file oscillations: an in vitro evaluation. J Endod 2010;36:880-3.

30. Verhaagen B. Acoustic streaming induced by ultrasonically oscillating endodontic files. In: Root Canal Cleaning through Cavitation and Microstreaming. PhD Thesis. University of Twente: Enschede, The Netherlands. 2012.

31. De Deus QD. Frequency, location, and direction of the lateral, secondary, and accessory canals. J Endod 1975;1:361-6.

32. Malki M, Verhaagen B, Jiang L-M, et al. Irrigant flow beyond the insertion depth of an ultrasonically oscillating file in straight and curved root canals: visualization and cleaning efficacy. J Endod 2012;38:657-61. 